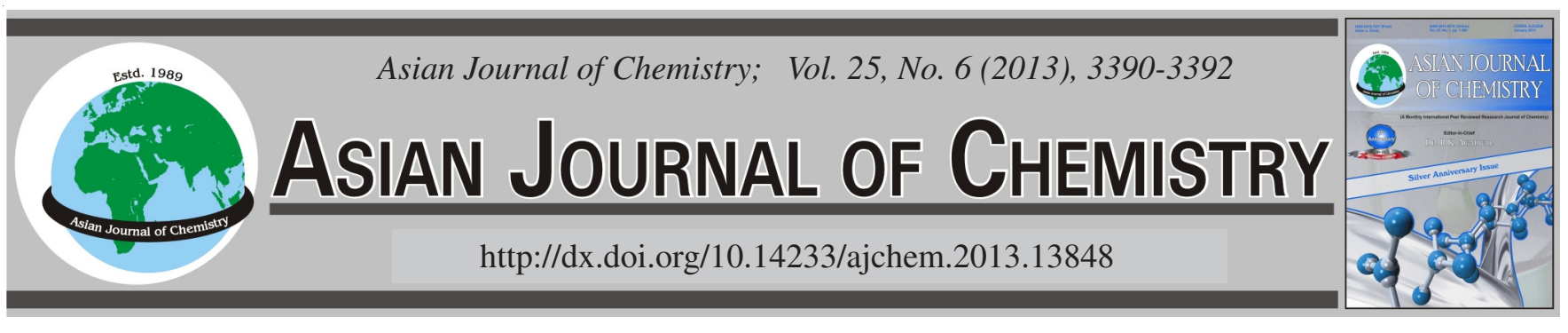

\title{
Green Synthesis of Schiff Bases Derived from 4,5-Diazafluorene-9-one in Aqueous Medium Under Microwave Irradiation
}

Hui CANG ${ }^{*}$, Yu Lu, WenYan Shi, Jingling ShaO and Qi Xu

Chemical and Biological Engineering College, Yancheng Institute of Technology, Yancheng 224051, Jiangsu Province, P.R. China

*Corresponding author: Fax: +86 515 88298618; Tel: +86 515 88298611; E-mail: canghui@ycit.edu.cn

(Received: 4 April 2012;

Accepted: 24 December 2012)

AJC-12595

A series of Schiff bases were successfully synthesized with satisfactory yield from various amines and 4,5-diazafluorene-9-one in water by using polystyrene sulfonic acid as a catalyst under microwave irradiation.

Key Words: Schiff base, 4,5-Diazafluorene-9-one, Microwave irradiation, Aqueous medium.

\section{INTRODUCTION}

4,5-Diazafluoren-9-one and their derivatives are also strong bidentate chelating and bridging agents ${ }^{1}$, which have been widely explored due to their easy accessibility, easy modification and relative air stability ${ }^{2}$. Synthesis of Schiff bases containing a bipyridine type metal receptor $^{3}$, has attracted considerable attention due to their many applications in the fields of catalyst $t^{4}$, conducting and photoresponsive materials ${ }^{5}$ and anion sensor ${ }^{6}$ etc. But, higher temperatures, increased reaction times, plenty of organic solvent and the use of a catalyst were generally required in previous synthesis of Schiff bases from 4,5-diazafluorene-9-one . $^{7,8}$.

In recent years, microwave irradiation was demonstrated to be a valuable tool in organic synthesis resulting in faster and cleaner reactions that sometimes exhibit different reactivates due to specific microwave absorption ${ }^{9}$. From an environmental view point, we report herein, for the first time, the reaction (Scheme-I) can be successfully accomplished with satisfactory yield in water by using polystyrene sulfonic acid (PSSA) as a catalyst under microwave irradiation (MW), which is a rapid and environmentally friendly preparation of schiff bases from 4,5-diazafluorene-9-one compared to conventional heating method.

\section{EXPERIMENTAL}

All reagents and solvents were purchased commercially as AC grade and were used without further purification unless noted. The molecular weight of polystyrene sulfonic acid is 70,000 . Organic solvents were dried over $4 \AA$ activated molecular sieves prior to use. 4,5-diazafluoren-9-one was obtained as light yellow needle by oxidation of 1,10-phenanthroline

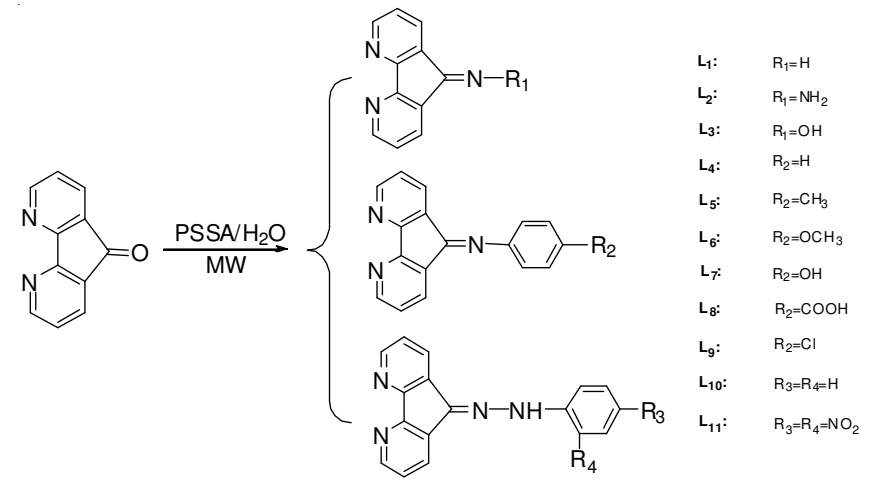

Scheme-I: Synthesis of schiff bases derived from 4,5-diazafluorene-9-one

with $\mathrm{KMnO}_{4}$ in a $\mathrm{KOH}$ solution following a literature method ${ }^{10}$. The microwave reaction was carried out in the WF-4000M closed type microwave synthesis system with the refluxing equipment. Melting points were determined in open capillaries and uncorrected. ${ }^{1} \mathrm{H}$ NMR spectra were obtained using Bruker DRX300 spectrometer with TMS. The IR spectra were determined as potassium bromide pellet on a Bruker Equinox55 FT-IR spectrophotometer. UV-VIS spectra were performed with a UV-2501PC spectrophotometer. Mass spectra and element analysis were done with LC-MSD-Trap-SL and Elementar Vario EL III.

General procedure for preparation of $L_{1}-L_{11}$ : All the Schiff bases derived from 4,5-diazafluorene-9-one were synthesized by modification of a literature procedure ${ }^{11}$. A mixture of 4,5-diazafluorene-9-one (455 mg, $2.5 \mathrm{mmol}$ ) and amines $(3 \mathrm{mmol}$ ) were dissolved in $20 \%$ polystyrene sulfonic acid solution in water $(5 \mathrm{~mL})$ and then were exposed to microwave irradiation $\left(700 \mathrm{~W}, 100^{\circ} \mathrm{C}\right)$ for $7 \mathrm{~min}$. The desired 
precipitate was filtered after cooling, washed with ethanol 3 times and dried in vacuum oven at $50{ }^{\circ} \mathrm{C}$. The results of yield, melt point and UV-visible spectra were listed in Table-1.

9-Imine-4,5-diazafluorene $\left(\mathbf{L}_{\mathbf{1}}\right)$ : ${ }^{1} \mathrm{H}$ NMR $\left(\mathrm{CDCl}_{3}, 300\right.$ MHz): $\delta 8.82\left(\mathrm{dd}, 2 \mathrm{H}, J_{1}=5.1, J_{2}=1.5 \mathrm{~Hz}\right.$, pyridyl $\mathrm{H}$ ortho to $\mathrm{N}), 8.05\left(\mathrm{dd}, 2 \mathrm{H}, J_{1}=5.1, J_{2}=1.5 \mathrm{~Hz}\right.$, pyridyl $\mathrm{H}$ para to $\left.\mathrm{N}\right)$, $7.38\left(\mathrm{t}, 2 \mathrm{H}, J_{1}=7.6, J_{2}=5.1 \mathrm{~Hz}\right.$, pyridyl H meta to $\left.\mathrm{N}\right), 5.45(\mathrm{~s}$, $1 \mathrm{H},=\mathrm{NH})$. IR $\left(\mathrm{KBr}, \nu_{\max }, \mathrm{cm}^{-1}\right): 3314,3058,1655,1590,1555$, 1420, 1200, 801, 726. Elemental analysis calcd. (\%) for $\mathrm{C}_{11} \mathrm{H}_{7} \mathrm{~N}_{3}$ : C, 72.92; H, 3.89; N, 23.19. Found (\%): C, 72.89; H, 3.92; N, 23.15. MS: $\mathrm{m} / \mathrm{z}=181.01$ (Calcd.: 181.06).

9-Hydrazone-4,5-diazafluorene $\left(\mathbf{L}_{2}\right)$ : ${ }^{1} \mathrm{H} \mathrm{NMR}\left(\mathrm{CDCl}_{3}\right.$, $300 \mathrm{MHz}$ ): $\delta 8.75$ (dd, $1 \mathrm{H}, J_{1}=5.1, J_{2}=1.6 \mathrm{~Hz}$, pyridyl $\mathrm{H}$ ortho to $\mathrm{N}$ ), 8.65 (dd, $1 \mathrm{H}, J_{1}=5.0, J_{2}=1.6 \mathrm{~Hz}$, pyridyl $\mathrm{H}$ ortho to $\mathrm{N}), 8.27$ (dd, $1 \mathrm{H}, J_{1}=7.7, J_{2}=1.6 \mathrm{~Hz}$, pyridyl $\mathrm{H}$ para to $\left.\mathrm{N}\right)$, $8.16\left(\mathrm{dd}, 1 \mathrm{H}, J_{1}=7.8, J_{2}=1.6 \mathrm{~Hz}\right.$, pyridyl $\mathrm{H}$ para to $\left.\mathrm{N}\right), 7.50$ $\left(\mathrm{t}, 1 \mathrm{H}, J_{1}=7.7, J_{2}=5.1 \mathrm{~Hz}\right.$, pyridyl H meta to $\left.\mathrm{N}\right), 7.39(\mathrm{t}, 1 \mathrm{H}$, $J_{1}=7.7, J_{2}=5.0 \mathrm{~Hz}$, pyridyl $\mathrm{H}$ meta to $\left.\mathrm{N}\right), 6.65\left(\mathrm{~s}, 2 \mathrm{H},-\mathrm{NH}_{2}\right)$. IR $\left(\mathrm{KBr}, \mathrm{v}_{\max }, \mathrm{cm}^{-1}\right): 3376,3320,3058,1646,1593,1561$, 1206, 799, 723. Elemental analysis calcd. (\%) for $\mathrm{C}_{12} \mathrm{H}_{9} \mathrm{~N}_{3}$ : C, 67.34; H, 4.11; N, 28.55. Found (\%): C, 67.30; H, 4.10; N, 28.52. MS: $\mathrm{m} / \mathrm{z}=196.01$ (calcd.: 196.06).

9-Oxime-4,5-diazafluorene $\left(\mathbf{L}_{3}\right)$ : Sodium carbonate $(0.016$ $\mathrm{g}, 1.5 \mathrm{mmol})$ was used as a neutralizer in the reaction. ${ }^{1} \mathrm{H}$ NMR $\left(\mathrm{CDCl}_{3}, 300 \mathrm{MHz}\right): \delta 13.14(\mathrm{~s}, 1 \mathrm{H},-\mathrm{OH}), 8.73\left(\mathrm{dd}, 1 \mathrm{H}, J_{1}=\right.$ $5.1, J_{2}=1.6 \mathrm{~Hz}$, pyridyl $\mathrm{H}$ ortho to $\left.\mathrm{N}\right), 8.67\left(\mathrm{dd}, 1 \mathrm{H}, J_{1}=5.0\right.$, $J_{2}=1.6 \mathrm{~Hz}$, pyridyl $\mathrm{H}$ ortho to $\left.\mathrm{N}\right), 8.27\left(\mathrm{dd}, 1 \mathrm{H}, J_{1}=7.7, J_{2}=\right.$ $1.6 \mathrm{~Hz}$, pyridyl $\mathrm{H}$ para to $\mathrm{N}), 8.17$ (dd, $1 \mathrm{H}, J_{1}=7.8, J_{2}=1.6$ $\mathrm{Hz}$, pyridyl $\mathrm{H}$ para to $\mathrm{N}), 7.53\left(\mathrm{t}, 1 \mathrm{H}, J_{1}=7.7, J_{2}=5.1 \mathrm{~Hz}\right.$, pyridyl $\mathrm{H}$ meta to N), 7.46 (t, $1 \mathrm{H}, J_{1}=7.7, J_{2}=5.0 \mathrm{~Hz}$, pyridyl $\mathrm{H}$ meta to N). IR (KBr, $\left.v_{\max }, \mathrm{cm}^{-1}\right): 3141,3036,1627,1593$, 1564, 1495, 1399, 1008, 949, 748. Elemental analysis calcd. (\%) for $\mathrm{C}_{11} \mathrm{H}_{7} \mathrm{~N}_{3} \mathrm{O}: \mathrm{C}, 67.00 ; \mathrm{H}, 3.58 ; \mathrm{N}, 21.31$. Found (\%): C, 69.92.; H, 3.56; N, 21.36. MS: m/z = 197.10 (calcd.: 197.06).

9-Anilino-4,5-diazafluorene $\left(\mathbf{L}_{4}\right)$ : ${ }^{1} \mathrm{H} \mathrm{NMR}\left(\mathrm{CDCl}_{3}, 300\right.$ $\mathrm{MHz}): \delta 8.81\left(\mathrm{dd}, 1 \mathrm{H}, J_{1}=4.9, J_{2}=1.5 \mathrm{~Hz}\right.$, pyridyl $\mathrm{H}$ ortho to $\mathrm{N}), 8.65\left(\mathrm{dd}, 1 \mathrm{H}, J_{1}=4.9, J_{2}=1.6 \mathrm{~Hz}\right.$, pyridyl $\mathrm{H}$ ortho to $\left.\mathrm{N}\right)$, $8.26\left(\mathrm{dd}, 1 \mathrm{H}, J_{1}=7.8, J_{2}=1.6 \mathrm{~Hz}\right.$, pyridyl H para to $\left.\mathrm{N}\right), 7.43$ $(\mathrm{m}, 3 \mathrm{H}$, pyridyl $\mathrm{H}$ meta to $\mathrm{N}$ and $\mathrm{Ar}-\mathrm{H}), 7.28\left(\mathrm{t}, 1 \mathrm{H}, J_{1}=7.7\right.$, $J_{2}=5.1 \mathrm{~Hz}$, pyridyl H meta to N), 7.02 (m, 3H, Ar-H), 6.87 (dd, $1 \mathrm{H}, J_{1}=7.8, J_{2}=1.5 \mathrm{~Hz}$, pyridyl H para to N). IR (KBr, $\left.V_{\max }, \mathrm{cm}^{-1}\right): 3045,1656,1592,1561,1401,748,710$. Elemental analysis calcd. (\%) for $\mathrm{C}_{17} \mathrm{H}_{11} \mathrm{~N}_{3}$ : C, 79.36; H, 4.31; N, 16.33 .
Found (\%): C, 79.30; H, 4.35; N, 16.36. MS: $\mathrm{m} / \mathrm{z}=257.13$ (calcd.: 257.10).

9-(4-Methyanilino)-4,5-diazafluorene $\left(\mathbf{L}_{5}\right)$ : ${ }^{1} \mathrm{H}$ NMR $\left(\mathrm{CDCl}_{3}, 300 \mathrm{MHz}\right): \delta 8.80\left(\mathrm{dd}, 1 \mathrm{H}, J_{1}=5.0, J_{2}=1.6 \mathrm{~Hz}\right.$, pyridyl $\mathrm{H}$ ortho to $\mathrm{N}), 8.64\left(\mathrm{dd}, 1 \mathrm{H}, J_{1}=4.9, J_{2}=1.6 \mathrm{~Hz}\right.$, pyridyl $\mathrm{H}$ ortho to $\mathrm{N}$ ), 8.25 (dd, $1 \mathrm{H}, J_{1}=7.6, J_{2}=1.5 \mathrm{~Hz}$, pyridyl H para to $\mathrm{N}$ ), 7.39 (t, $1 \mathrm{H}, J_{1}=7.6, J_{2}=5.1 \mathrm{~Hz}$, pyridyl $\mathrm{H}$ meta to $\mathrm{N}$ ), 7.25 (m, 2H, pyridyl H meta to $\mathrm{N}$ and Ar-H), 6.98 (m, 2H, pyridyl $\mathrm{H}$ para to $\mathrm{N}$ and $\mathrm{Ar}-\mathrm{H}), 6.90(\mathrm{~d}, 2 \mathrm{H}, J=8.2 \mathrm{~Hz}, \mathrm{Ar}-\mathrm{H})$, $2.43\left(\mathrm{~s}, 3 \mathrm{H},-\mathrm{CH}_{3}\right)$. IR ( $\left.\mathrm{KBr}, \mathrm{v}_{\max }, \mathrm{cm}^{-1}\right)$ : 3046, 2954, 2869, 1642, 1590, 1557, 1399, 752, 729. Elemental analysis calcd. (\%) for $\mathrm{C}_{18} \mathrm{H}_{13} \mathrm{~N}_{3}: \mathrm{C}, 79.68 ; \mathrm{H}, 4.83 ; \mathrm{N}, 15.49$. Found (\%): C, 79.62; H, 4.85; N, 15.46. MS: $\mathrm{m} / \mathrm{z}=271.13$ (calcd.: 271.11).

9-(4-Methoxyanilino)-4,5-diazafluorene $\left(\mathbf{L}_{6}\right)$ : ${ }^{1} \mathrm{H}$ NMR $\left(\mathrm{CDCl}_{3}, 300 \mathrm{MHz}\right): \delta 8.80\left(\mathrm{dd}, 1 \mathrm{H}, J_{1}=5.0, J_{2}=1.6 \mathrm{~Hz}\right.$, pyridyl $\mathrm{H}$ ortho to $\mathrm{N}), 8.65\left(\mathrm{dd}, 1 \mathrm{H}, J_{1}=5.0, J_{2}=1.6 \mathrm{~Hz}\right.$, pyridyl $\mathrm{H}$ ortho to $\mathrm{N}), 8.23\left(\mathrm{dd}, 1 \mathrm{H}, J_{1}=7.7, J_{2}=1.5 \mathrm{~Hz}\right.$, pyridyl $\mathrm{H}$ para to $\mathrm{N}$ ), $7.38\left(\mathrm{t}, 1 \mathrm{H}, J_{1}=7.7, J_{2}=5.0 \mathrm{~Hz}\right.$, pyridyl $\mathrm{H}$ meta to $\mathrm{N}$ ), $7.09\left(\mathrm{dd}, 1 \mathrm{H}, J_{1}=7.8, J_{2}=1.7 \mathrm{~Hz}\right.$, pyridyl $\mathrm{H}$ para to $\mathrm{N}), 6.98$ (m, 5H, pyridyl $\mathrm{H}$ meta to $\mathrm{N}$ and Ar-H), 3.92 $\left(\mathrm{s}, 3 \mathrm{H},-\mathrm{OCH}_{3}\right)$. IR $\left(\mathrm{KBr}, \mathrm{v}_{\max }, \mathrm{cm}^{-1}\right)$ : 3043, 2999, 2837, 1634, 1590, 1559, 1497, 1400, 1234, 1023, 845, 757. Elemental analysis calcd. (\%) for $\mathrm{C}_{18} \mathrm{H}_{13} \mathrm{~N}_{3} \mathrm{O}: \mathrm{C}, 75.25 ; \mathrm{H}, 4.56 ; \mathrm{N}, 13.95$. Found (\%): C, 75.20; H, 4.58; N, 13.97. MS: $\mathrm{m} / \mathrm{z}=287.07$ (calcd.: 287.11).

9-(4-Hydroxyanilino)-4,5-diazafluorene $\left(\mathrm{L}_{7}\right):{ }^{1} \mathrm{H} \mathrm{NMR}$ $\left(\mathrm{CDCl}_{3}, 300 \mathrm{MHz}\right): \delta 9.53(\mathrm{~s}, 1 \mathrm{H},-\mathrm{OH}), 8.79\left(\mathrm{dd}, 1 \mathrm{H}, J_{1}=\right.$ $4.9, J_{2}=1.5 \mathrm{~Hz}$, pyridyl $\mathrm{H}$ ortho to $\left.\mathrm{N}\right), 8.67\left(\mathrm{dd}, 1 \mathrm{H}, J_{1}=4.9\right.$, $J_{2}=1.5 \mathrm{~Hz}$, pyridyl $\mathrm{H}$ ortho to $\left.\mathrm{N}\right), 8.27\left(\mathrm{dd}, 1 \mathrm{H}, J_{1}=7.6, J_{2}=\right.$ $1.5 \mathrm{~Hz}$, pyridyl H para to $\mathrm{N}), 7.54\left(\mathrm{t}, 1 \mathrm{H}, J_{1}=7.6, J_{2}=4.9 \mathrm{~Hz}\right.$, pyridyl $\mathrm{H}$ meta to $\mathrm{N}$ ), 7.27 (t, $1 \mathrm{H}, J_{1}=7.6, J_{2}=4.9 \mathrm{~Hz}$, pyridyl $\mathrm{H}$ meta to $\mathrm{N}$ ), 7.13 (dd, $1 \mathrm{H}, J_{1}=7.6, J_{2}=1.5 \mathrm{~Hz}$, pyridyl $\mathrm{H}$ para to $\mathrm{N}), 6.90(\mathrm{~m}, 4 \mathrm{H}, \mathrm{Ar}-\mathrm{H})$. IR $\left(\mathrm{KBr}, \mathrm{v}_{\max }, \mathrm{cm}^{-1}\right)$ : 3144 , 3037, 1638, 1592, 1561, 1504, 1401, 1276, 1230, 1165, 758, 708. Elemental analysis calcd. (\%) for $\mathrm{C}_{17} \mathrm{H}_{11} \mathrm{~N}_{3} \mathrm{O}: \mathrm{C}, 74.71$; H, 4.06; N, 15.38. Found (\%): C, 74.65; H, 4.00; N, 15.42 . MS: $\mathrm{m} / \mathrm{z}=272.85$ (calcd.: 273.09).

9-(4-Carboxyanilino)-4,5-diazafluorene $\left(\mathrm{L}_{8}\right)$ : ${ }^{1} \mathrm{H}$ NMR $\left(\mathrm{CDCl}_{3}, 300 \mathrm{MHz}\right): \delta 12.93(\mathrm{~s}, 1 \mathrm{H},-\mathrm{COOH}), 8.82\left(\mathrm{dd}, 1 \mathrm{H}, J_{1}\right.$ $=4.9, J_{2}=1.5 \mathrm{~Hz}$, pyridyl $\mathrm{H}$ ortho to $\left.\mathrm{N}\right), 8.68\left(\mathrm{dd}, 1 \mathrm{H}, J_{1}=\right.$ $4.9, J_{2}=1.5 \mathrm{~Hz}$, pyridyl $\mathrm{H}$ ortho to $\left.\mathrm{N}\right), 8.29\left(\mathrm{dd}, 1 \mathrm{H}, J_{1}=7.7\right.$, $J_{2}=1.5 \mathrm{~Hz}$, pyridyl H para to N), $8.02(\mathrm{~d}, 2 \mathrm{H}, J=8.6 \mathrm{~Hz}, \mathrm{Ar}-$ $\mathrm{H}), 7.56\left(\mathrm{t}, 1 \mathrm{H}, J_{1}=7.7, J_{2}=4.9 \mathrm{~Hz}\right.$, pyridyl $\mathrm{H}$ meta to $\left.\mathrm{N}\right)$,

TABLE-1

RESULTS OF YIELD, MELTING POINT AND UV-VISIBLE SPECTRA OF $\mathbf{L}_{1-11}$

\begin{tabular}{cccl}
\hline No. & Yield $(\%)$ & m.p. $\left({ }^{\circ} \mathrm{C}\right)$ & \multicolumn{1}{c}{ UV-VIS $\lambda_{\max }\left(>235 \mathrm{~nm}, \varepsilon \times 10^{4}\right)^{\mathrm{a}}$} \\
\hline $\mathbf{L}_{\mathbf{1}}$ & 78 & $195-196$ & $249(2.40), 304(0.99), 317(1.01)$. \\
$\mathbf{L}_{\mathbf{2}}$ & 82 & $208-210$ & $245(2.45), 303(0.98), 313(1.13)$. \\
$\mathbf{L}_{\mathbf{3}}$ & 80 & $>300$ & $242(2.41), 308(1.00), 315(0.96)$. \\
$\mathbf{L}_{\mathbf{4}}$ & 87 & $188-189$ & $240(2.40), 302(0.95), 315(1.02)$. \\
$\mathbf{L}_{\mathbf{5}}$ & 89 & $225-226$ & $244(2.56,302(0.98), 314(1.13), 416(0.20)$. \\
$\mathbf{L}_{\mathbf{6}}$ & 85 & $202-203$. & $252(3.80), 300(1.44), 314(1.04), 435(0.29)$. \\
$\mathbf{L}_{\mathbf{7}}$ & 90 & $>300$ & $244(2.69), 302(1.15), 314(0.97), 433(0.26)$ \\
$\mathbf{L}_{\mathbf{8}}$ & 81 & $>300$ & $245(2.73), 265(2.51), 302(1.01), 314(0.91), 394(0.16)$ \\
$\mathbf{L}_{\mathbf{9}}$ & 93 & $216-217$ & $246(3.24), 302(1.15), 314(1.01), 405(0.20)$ \\
$\mathbf{L}_{\mathbf{1 0}}$ & 85 & $188-189$ & $242(2.03), 303(1.22), 314(1.18), 396(2.50)$ \\
$\mathbf{L}_{\mathbf{1 1}}$ & 83 & $>300$ & $\mathrm{CH}_{3} \mathrm{CN}: 303(0.58), 385(1.30), 406(1.27), 562(0.32)$ \\
\hline
\end{tabular}

${ }^{\mathrm{a}} \mathrm{L}_{1}-\mathrm{L}_{10}$ in $\mathrm{CHCl}_{3}$ and $\mathrm{L}_{11}$ in $\mathrm{CH}_{3} \mathrm{CN}$. 
$7.24\left(\mathrm{t}, 1 \mathrm{H}, J_{1}=7.7, J_{2}=4.9 \mathrm{~Hz}\right.$, pyridyl H meta to $\left.\mathrm{N}\right), 7.17$ $(\mathrm{d}, 2 \mathrm{H}, J=8.6 \mathrm{~Hz}, \mathrm{Ar}-\mathrm{H}), 6.83\left(\mathrm{dd}, 1 \mathrm{H}, J_{1}=7.7, J_{2}=1.5 \mathrm{~Hz}\right.$, pyridyl $\mathrm{H}$ para to $\mathrm{N})$. IR $\left(\mathrm{KBr}, \nu_{\max }, \mathrm{cm}^{-1}\right)$ : 3098, 3032, 1702, 1702, 1663, 1598, 1566, 1398, 1259, 1164, 752, 723. Elemental analysis: calcd. (\%) for $\mathrm{C}_{18} \mathrm{H}_{11} \mathrm{~N}_{3} \mathrm{O}_{2}$ : C, 71.75; H, 3.68; N, 13.95. Found (\%): C, 71.70; H, 3.63; N, 13.89. MS: m/z = 300.99 (calcd.: 301.09).

9-(4-Chloroanilino)-4,5-diazafluorene $\left(\mathbf{L}_{9}\right)$ : ${ }^{1} \mathrm{H}$ NMR $\left(\mathrm{CDCl}_{3}, 300 \mathrm{MHz}\right): \delta 8.83\left(\mathrm{dd}, 1 \mathrm{H}, J_{1}=5.0, J_{2}=1.5 \mathrm{~Hz}\right.$, pyridyl $\mathrm{H}$ ortho to $\mathrm{N}), 8.70\left(\mathrm{dd}, 1 \mathrm{H}, J_{1}=5.0, J_{2}=1.5 \mathrm{~Hz}\right.$, pyridyl $\mathrm{H}$ ortho to $\mathrm{N}$ ), $8.26\left(\mathrm{dd}, 1 \mathrm{H}, J_{1}=7.6, J_{2}=1.5 \mathrm{~Hz}\right.$, pyridyl $\mathrm{H}$ para to $\mathrm{N}$ ), 7.43 (m, 3H, pyridyl $\mathrm{H}$ meta to $\mathrm{N}$ and Ar-H), 7.07 (t, 1H, $J_{1}=7.7, J_{2}=5.0 \mathrm{~Hz}$, pyridyl $\mathrm{H}$ meta to $\mathrm{N}$ ), $7.03\left(\mathrm{dd}, 1 \mathrm{H}, J_{1}=7.8, J_{2}=1.5 \mathrm{~Hz}\right.$, pyridyl H para to $\left.\mathrm{N}\right), 6.95$ $(\mathrm{d}, 2 \mathrm{H}, J=8.2 \mathrm{~Hz}, \mathrm{Ar}-\mathrm{H})$. IR (KBr, $\left.v_{\max }, \mathrm{cm}^{-1}\right): 3046,1656$, 1592, 1561, 1400, 1087, 839, 749, 730. Elemental analysis calcd. (\%) for $\mathrm{C}_{18} \mathrm{H}_{10} \mathrm{~N}_{3} \mathrm{Cl}$ : C, 69.99; H, 3.45; N, 14.40. Found (\%): C, 69.93; H, 3.46; N, 14.45. MS: $\mathrm{m} / \mathrm{z}=291.01$ (calcd.: 291.06).

9-Phenylhydrazone-4,5-diazafluorene $\left(\mathbf{L}_{10}\right)$ : ${ }^{1} \mathrm{H}$ NMR (DMSO, $300 \mathrm{MHz}): \delta 10.72$ (s, 1H, -NH), 8.90 (d, 1H, $J=7.7$ $\mathrm{Hz}$, pyridyl $\mathrm{H}$ para to $\mathrm{N}$ ), 8.70 (dd, $2 \mathrm{H}, J=4.6 \mathrm{~Hz}$, pyridyl $\mathrm{H}$ ortho to $\mathrm{N}$ ), 8.29 (dd, $1 \mathrm{H}, J=7.7 \mathrm{~Hz}$, pyridyl $\mathrm{H}$ para to $\mathrm{N}$ ), 7.59 (m, 3H, pyridyl $\mathrm{H}$ meta to $\mathrm{N}$ and Ar-H), 7.45 (m, 3H, pyridyl $\mathrm{H}$ meta to $\mathrm{N}$ and Ar-H), 7.10 (m, 1H, Ar-H). IR (KBr, $\left.\mathrm{n}_{\max }, \mathrm{cm}^{-1}\right): 3385,3047,1652,1591,1560,1404,750,713$. Elemental analysis: calcd. (\%) for $\mathrm{C}_{17} \mathrm{H}_{12} \mathrm{~N}_{4}$ : C, 74.98; H, 4.44; $\mathrm{N}, 20.58$. Found (\%): C, 74.92; H, 4.41; N, 20.64. MS: m/z = 272.14 (calcd.: 272.11).

9-(2,4-Dinitrophenylhydrazone)-4,5-diazafluorene $\left(\mathbf{L}_{11}\right):{ }^{1} \mathrm{H}$ NMR (DMSO, $\left.300 \mathrm{MHz}\right): \delta 12.01(\mathrm{~s}, 1 \mathrm{H},-\mathrm{NH})$, $8.96(\mathrm{~s}, 1 \mathrm{H}, \mathrm{Ar}-\mathrm{H}), 8.84$ (d, $1 \mathrm{H}, J=5.0 \mathrm{~Hz}$, pyridyl $\mathrm{H}$ ortho to $\mathrm{N}), 8.76(\mathrm{~d}, 1 \mathrm{H}, J=4.9, \mathrm{~Hz}$, pyridyl $\mathrm{H}$ ortho to $\mathrm{N}), 8.59$ (d, $1 \mathrm{H}, J=7.8 \mathrm{~Hz}$, pyridyl $\mathrm{H}$ para to $\mathrm{N}), 8.53(\mathrm{~d}, 1 \mathrm{H}, J=7.8 \mathrm{~Hz}$, pyridyl $\mathrm{H}$ para to $\mathrm{N}), 8.42(\mathrm{~m}, 2 \mathrm{H}, \mathrm{Ar}-\mathrm{H}), 7.73$ (t, $1 \mathrm{H}, J_{1}=$ $7.7, J_{2}=5.0 \mathrm{~Hz}$, pyridyl H meta to $\left.\mathrm{N}\right), 7.56\left(\mathrm{t}, 1 \mathrm{H}, J_{1}=7.8, J_{2}\right.$ $=5.0 \mathrm{~Hz}$, pyridyl H meta to N). IR $\left(\mathrm{KBr}, v_{\max }, \mathrm{cm}^{-1}\right): 3414$,
3100, 1616, 1594, 1557, 1496, 1404, 1334, 1095, 754, 740, $\lambda_{\max }(>235 \mathrm{~nm}, \varepsilon)$ : Elemental analysis: calcd. (\%) for $\mathrm{C}_{17} \mathrm{H}_{10} \mathrm{~N}_{6} \mathrm{O}_{4}$ : C, 56.36; H, 2.78; N, 23.20. Found. (\%): C, 56.32; H, 2.74; N, 23.25. MS: $\mathrm{m} / \mathrm{z}=362.14$ (calcd.: 362.08).

\section{Conclusion}

This work has demonstrated an efficient and general microwave irradiation protocol for the synthesis of schiff bases derived from 4,5-diazafluorene-9-one under microwave irradiation using polystyrene sulfonic acid as a catalyst in aqueous medium. The simple product isolation via filtration precludes the use of a organic solvent thus culminating in an environmentally benign aqueous protocol for the synthesis of schiff bases derived from 4,5-diazafluorene-9-one.

\section{ACKNOWLEDGEMENTS}

This work was funded by the Applied Basic Research Project of Yancheng Institute of Technology of Chian, Jiangsu (Grant No. XKR2011005).

\section{REFERENCES}

1. D.A.V. Griend, D.K. Bediako, M.J. Devries, A.D. Nathan and P.H. Lee, Inorg. Chem., 47, 656 (2008).

2. Y.X. Wang, W. Perez, G.Y. Zheng, D.P. Rillema and C.L. Huber, Inorg. Chem., 37, 2227 (1998).

3. A.L. Eckermann, K.D. Barker, M.R. Hartings, M.A. Ratner and T.J. Meade, J. Am. Chem. Soc., 127, 11880 (2005).

4. J. Hou, W.H. Sun, D. Zhang, L. Chen, W. Li, D. Zhao and H. Song, J. Mol. Catal. A: Chem., 231, 221 (2005).

5. C.Y. Duan, Z.L. Lu, X.Z. You, Z.Y. Zhou, T.C.W. Mak, Q. Luo and J.Y. Zhou, Polyhedron, 17, 4131 (1998).

6. Z.H. Lin, Y.G. Zhao, C.Y. Duan, B.G. Zhang and Z.P. Bai, J. Chem. Soc. Dalton Trans., 3678 (2006).

7. H. Cang, S.Q. Wang, W.Z. Yang and J.T. Wang, J. Chem. Res., 2009, 101 (2009).

8. Y. Wang and D.P. Rillema, Tetrahedron, 53, 12377 (1997).

9. C.O. Kappe, Angew. Chem. Int. Ed., 43, 6250 (2004).

10. L.J. Henderson, F.R. Fronczek Jr. and W.R. Cherry, J. Am. Chem. Soc., 106, 5876 (1984).

11. V. Polshettiwar and R.S. Varma, Tetrahedron Lett., 48, 5649 (2007). 